\title{
Independent respiratory navigators for improved 3D PSIR imaging of myocardial infarctions
}

\author{
Sangjune L Lee ${ }^{1}$, Michael Schär ${ }^{2}$, Sebastian Kozerke ${ }^{3}$, Ahmed A Harouni ${ }^{4}$, Valeria Sena-Weltin ${ }^{5}$, \\ M Muz Zviman ${ }^{5}$, Henry Halperin ${ }^{5}$, Elliot R McVeigh', Daniel A Herzka ${ }^{1 *}$
}

From 2011 SCMR/Euro CMR Joint Scientific Sessions

Nice, France. 3-6 February 2011

\section{Introduction}

The distribution of viable and infarcted myocardium is typically visualized using inversion recovery (IR) late gadolinium enhancement (1) or phase-sensitive inversion recovery (PSIR) sequences (2). Transitioning PSIR from breath-hold 2D to respiratory navigator-gated 3D imaging promises higher SNR and CNR, and whole heart coverage $(3,4)$. However, the optimal method for motion compensation with 3D PSIR is undetermined. With standard PSIR, the IR-prepared volume (first heartbeat) is corrected with the phase from the reference volume (second heartbeat), (Fig 1a). Current implementations of respiratory navigated 3D PSIR accept data for the reference based solely on navigator NAV1, which takes place over a heartbeat in advance. Respiratory motion occuring between NAV1 and reference volume acquisition, potentially corrupts reference image quality and may compromise the
PSIR image. We propose an independently navigated PSIR (INPSIR) sequence with a separate navigator, NAV2, dedicated to motion compensation of the reference volume (Fig 1b).

\section{Methods}

Imaging was performed on a 3T system (Philips Healthcare, Best, The Netherlands) using a 32-channel cardiac array coil (InVivo, Gainsville FL). Phantoms: agar gel phantoms with varying $T_{1}$ values were placed on a pneumatic motor that oscillated $20 \mathrm{~mm}$ in the anteroposterior direction every second. Animals: Under ACUC-approved protocol, six swine underwent $2 \mathrm{hr}$ LAD occlusion. Imaging took place 4-25 weeks postinfarction and 10-3 min post double-dose injection of Magnevist (Schering, Germany). A 3D GRE sequence with $1.25 \times 1.25 \times 4 \mathrm{~mm}^{3}$ resolution and 2.5 min scan time ( assuming 100\% gating efficiency) was used.

a) Standard PSIR

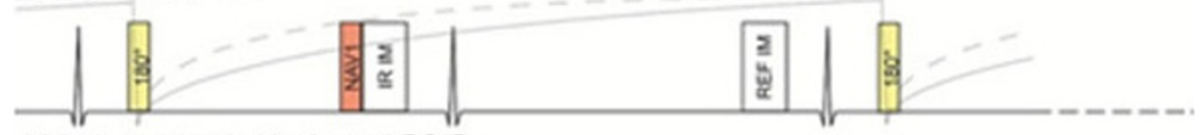

b) Independently Navigated PSIR

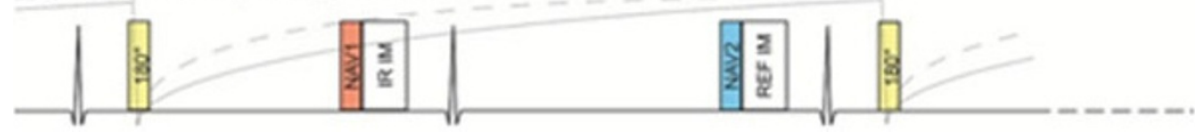

Figure 1 Schematic of PSIR and INPSIR acquisitions. The second independent navigator provides motion compensation for the phase reference images.

'Department of Biomedical Engineering, Johns Hopkins University School of

Medicine, Baltimore, MD, USA

Full list of author information is available at the end of the article

(c) 2011 Lee et al; licensee BioMed Central Ltd. This is an open access article distributed under the terms of the Creative Commons 


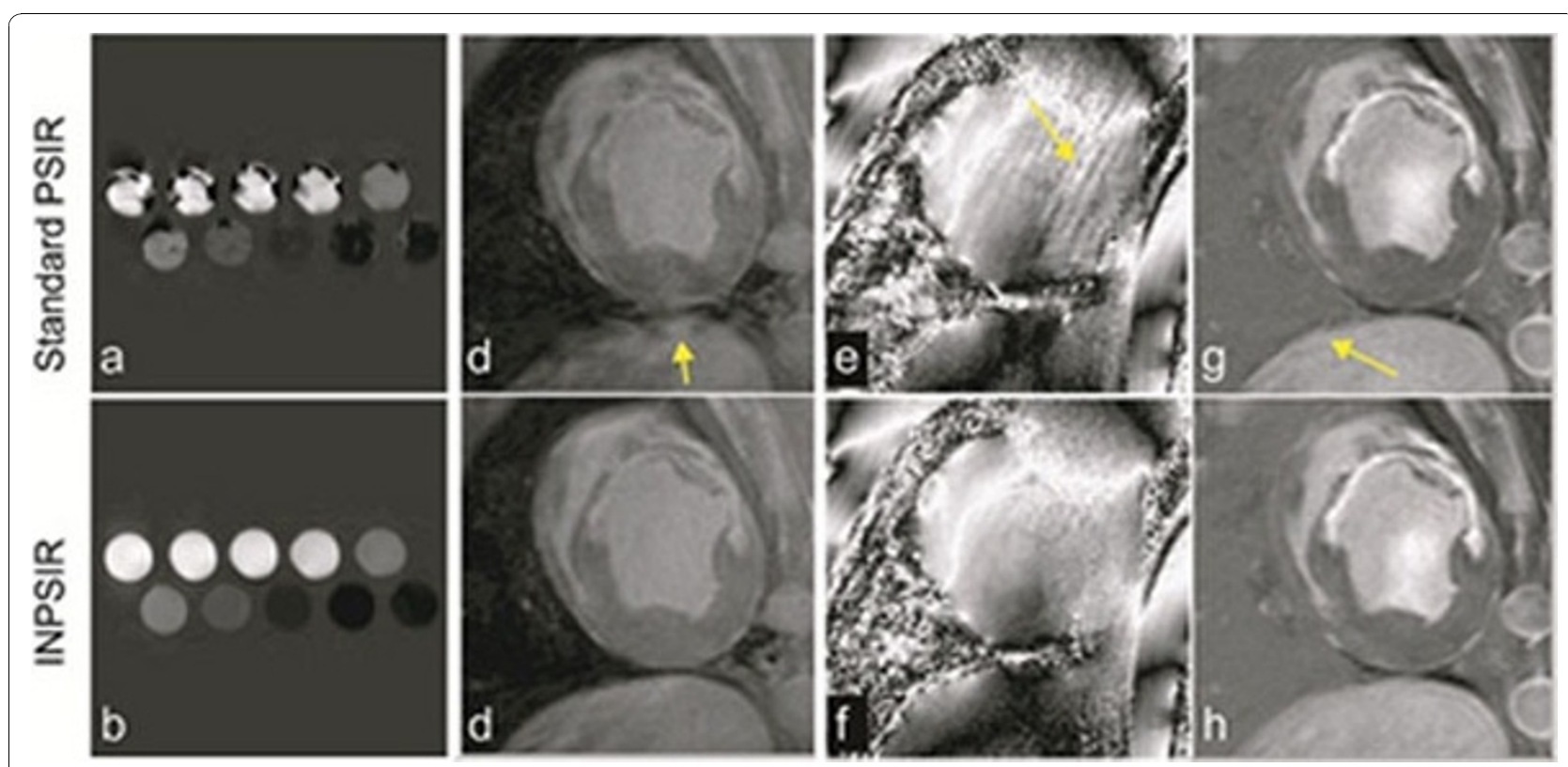

Figure 2 Comparison of images from PSIR and INPSIR: agar gels, reference image, single-coil phase reference, and phase-sensitive reconstructions. Note artifacts on phase image as well as lung-liver sharpness on INSPIR (arrows).

\section{Results}

Figure 1 (c-j) compares PSIR and INPSIR. (c-d) images from moving agar gels demonstrate possible artifacts when no motion compensation is used for the reference images. Short-axis images from an infarcted swine model including reference image magnitude, single-coil phase and phase-sensitive reconstruction are shown. The phase reference image was improved with INPSIR (arrows) and navigator efficiency was not affected.

\section{Conclusions}

INPSIR allows for improved reference images, uncorrupted by respiratory motion with no additional penalties in navigator efficiency. The lung-liver-heart boundaries were all sharper with INPSIR. We expect INPSIR will have a greater effect when using parallel imaging and may help obtain additional useful contrasts from phase reference images (5).

\footnotetext{
Author details

'Department of Biomedical Engineering, Johns Hopkins University School of Medicine, Baltimore, MD, USA. 'Philips Healthcare, Cleveland, OH, USA. ${ }^{3}$ Institute for Biomedical Engineering, University and ETH Zurich, Zurich, Switzerland. ${ }^{4}$ Department of Electrical and Computer Engineering, Johns Hopkins University, Baltimore, MD, USA. ${ }^{5}$ Division of Cardiology, Johns Hopkins University School of Medicine, Baltimore, MD, USA.
}

Published: 2 February 2011
doi:10.1186/1532-429X-13-S1-P18

Cite this article as: Lee et al:: Independent respiratory navigators for improved 3D PSIR imaging of myocardial infarctions. Journal of Cardiovascular Magnetic Resonance 2011 13(Suppl 1):P18.

\section{Submit your next manuscript to BioMed Central and take full advantage of:}

- Convenient online submission

- Thorough peer review

- No space constraints or color figure charges

- Immediate publication on acceptance

- Inclusion in PubMed, CAS, Scopus and Google Scholar

- Research which is freely available for redistribution
C Biomed Central 